\title{
Nature Inspired Techniques For Interference Management In Femtocells : A Survey
}

\author{
Shradha Deshmukh \\ G.H.Raisoni College of Engineering \\ Nagpur, India \\ Email: shradha.m.deshmukh@gmail.com
}

\author{
Mangala Madankar \\ G.H.Raisoni College of Engineering \\ Nagpur, India \\ Email: mangala.madankar@raisoni.net
}

\begin{abstract}
In the wireless communication system the transmitter and receiver close to each other to improve the data rates and capacity. Therefore, the wireless networks are more popular than the traditional wired services. In the wireless networks, to cover cells the low power nodes such as macrocells, picocells, femtocells base stations (BSs) deployed to improve the indoor coverage. The femtocell base station reduces operators operational cost, maintainance and infrastructure. At the time of femtocell deployment, the femtocell base station deal with a number of technical challenges, among those all the interference management is more important. In femtocell network, one femtocell creates the interference to its neighboring femtocells. To deal with interference management challenge number of researchers have suggested different types of solutions. The survey shows that nature inspired metaheuristic algorithm has the powerful impact on interference cancellation and avoidance. This survey paper focuses on bat algorithm for the resource allocation problem in a femtocell.
\end{abstract}

Index Terms-Femtocell, Interference Management, Bat Algorithm, Differential Evolution.

\section{INTRODUCTION}

The growth in the mobile device users the data traffic demands will be automatically increased, indirectly network operators will also have to significantly increase the capacity of networks. For this cellular network, the small cells have a number of nodes with low power such as macrocells, picocells, femtocells base stations (BSs) and these cells are combined in only one architecture called heterogeneous networks (HetNet). In that way, area spectral efficiency enhanced due to reduced distance in between transmitter and receiver. In heterogeneous network (HetNet) deployment provides coverage in such manner to cover the dead that time think out the transmitter not cause interference to users or user equipment's (UEs) by transmitting high power signals. By providing large number of channel per cell can improve the networking capacity.This means that adding number of channel per cells reduces the actual area of cells and thus increases the channel reuse. Drawback of outdoor cells who serving for indoor users such as: Indoor users require high power from an outdoor base station but base station fails to serve to the indoor users, Lower channel conditions of outdoor network cause the lower data rates for indoor users and the less number of outdoor BS in the densely populated area which raises the dead zones. To solve above all problems the indoor solution is Distributed Antenna System (DAS) and picocells. But these both solutions are not cost effective for some indoor users. A recent indoor solution is a femtocell, it can be easily deployed by users without any operator. Femtocells, also for indoor small coverage areas such as home base station and it is known as Femtocell Base Station (FBS) and it is connected the mobile user to network operator via wired and wireless technologies. Indoor base station consist of two different tiers or sometimes is called layers. The first tier is macrocell tier and femtocell tier, this architecture is known as two-tier architecture. The unplanned and random deployment of femtocells are in the femtocell tier. This has the advantage of capacity and coverage. The femtocell uses the same frequency band as of macrocells for better capacity. Because of this situation, the interference problem arises.

\section{Interference Management In Femtocell}

In this section, the different two types of interference faced by two-tier network are explained and detail about uplink interference and downlink interference problem are also explained. The femtocells are deployed in mainly CDMA and OFDMA technologies, so also briefly explained the problem faced by these two technologies.Femtocell has the challenge in the case of dense deployment tracking the neighbors and handover the user is not possible. Femtocells deployed in ad-hoc manner. So it is diffficult to manage the neighboring femtocells. Self-Configuration: Femtocell deployed by users and any time they get on and off hence the deployment of the femtocell is completely random and unplanned. Self-Configuration: Femtocell can configure itself whenever situation needed to add and remove user or location of femtocells get changed. The deployment of an indoor base station has two layers or tiers, conventional macrocell network is one of network and the second network is femtocell network. The femtocells are deployed in the Macrocell network in co-channel manner, suing the same frequency band. In this network, the interference problem is faced by both uplink and downlink.

\section{A. Types of Interference}

\section{1) Co-tier Interference}

Co-tier interference caused by the network element that belongs to the same tier or layer. To establish 


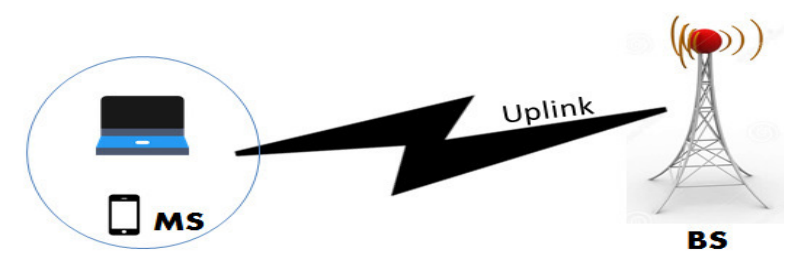

Fig.1 Uplink Transmission

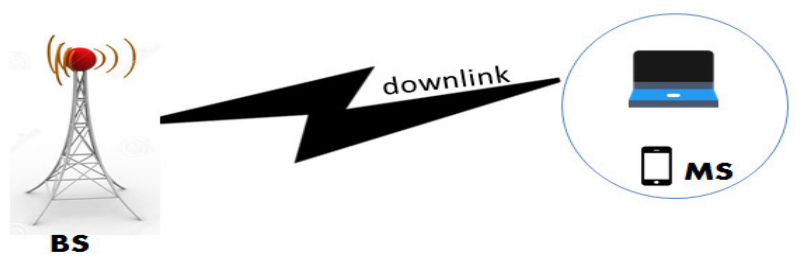

Fig.2 Downlink Transmission

communication link it would depend on the SINR, if SINR is higher than the threshold value then there is a communication gap and this gap is called Dead zone. Close access mode has major issue of co-tier interference as compare to open access mode. Femtocell user equipment(FUE) is the main cause of co-tier interference. In CDMA, the neighboring FUE is transmitting more power it will causes interference to the respective femtocell performance. In this situation, FBS states the power limits on FUE. In OFDMA, FBS sense the surrounding environment for sub channels depending on the quality of service(QOS) the FUE require a number of sub-channels. Then FAP allocates sub channels that address the lower level of interference. The downlink interference is caused by Femtocell Access Point (FAP), it causes the interference to the neighboring FUE. Due to close access of femtocells, the power exudes through doors, balconies, windows. From this, the exude power causes the interference to the neighboring FUE. In CDMA, the downlink interference creates dead zones. $3 \mathrm{G}$ system uses adaptive power control techniques at FAP to avoid above kind of interference. In OFDMA, if the allocation of sub-channels is different for each and every user in femtocell then the interference would be avoided.

2) ii. Cross-tier Interference

The element which belongs to different tier or layer which causes the cross-tier interference. For example, In downlink FBS will cause interference to neighboring MUE and in uplink interference the MUE will cause interference to its neighboring FBS. Macrocell indoor users interfered by the neighboring femtocells. Normally the femtocells are isolated, due to wall pentration, but sometimes the MUE creates sufficient interference to the femtocells. When the FUE act as a source of interference to MBS then the uplink cross-tier interference occur[5]. In CDMA, power control used to control the FUE from generating interference to the MBS. When MUE transmits high power to the nearer femtocells this creates interference to the FBS[5]. In the next case, FBS sense environment and do not ask for high power.
Sometimes for MUE, closed access mode area of femtocells are dead zones.

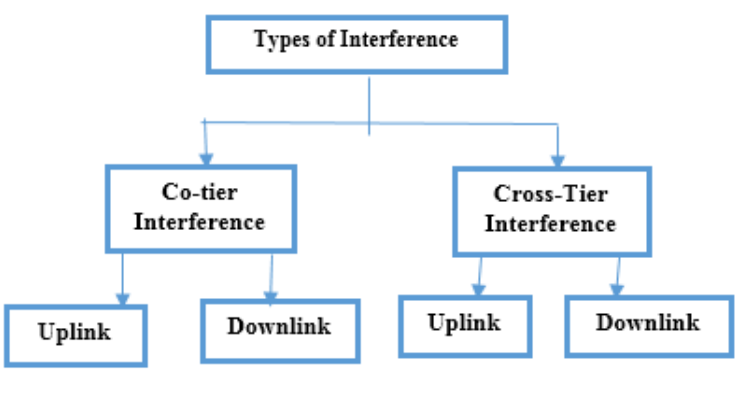

Fig.3 Types of interference

In CDMA, adaptive power control is needed because every time the changing environment is there. When FUE is close to a window having indoor station FAP, automatically FUE connects to MBS instead of FAP. In OFDMA, if MUE uses the different sub-channles then FBS do not cause downlink interference to MUE.

\section{B. Interference Management Techniques}

The technologies use many techniques to handle the cotier and cross-tier interference. Many schemes have been proposed by the researcher and also research is still going on. Some main technique would be summarized in this section. Nature Inspired Algorithms introduced the different classes of methods: those that develop problem-solving techniques from nature and those whose employ natural materials to determine solutions. Nature inspired algorithm has developed to solve the optimization problems. Following are two concepts in nature inspired computation: The Evolutionary Algorithm (EA) and the Swarm Intelligence Algorithm (SIA).

1) Evolutionary Algorithm (EA):

EA are optimization techniques and subset of evolutionary computations based on Darwin's principle. This principle says that in nature the fittest individual has the greater chance to survive. Following are the disciplines of EA: Genetic programming, Genetic algorithm, Evolution strategies, Evolutionary programming, and Differential evolution. All the algorithm has been developing to share the same characteristics such as selection operators, variation operators while solving the optimization problemGenetic Algorithm (GA) is used to reduce the coverage hole problems and balance user's power distribution. Genetic Algorithm(GA) has been mainly used in the macrocellfemtocell networks and also to increase coverage and reduce femtocell-femtocell interference. The work in [16] showed that the GA performed better at satisfying QOS in OFDMA system. GA proved to be a good candidate to solve resource allocation problem and advantage of a faster optimization process.

2) Swarm Intelligence Algorithm(SIA):

Swarm Intelligence Algorithm (SIA) introduced by Being in 1989. SIA is a collection behavior of self-organizes and decentralized system. Swarm intelligence applied on 
Table.1 : Comparison of Di erent Interference Management Technique

\begin{tabular}{|c|c|c|c|c|c|}
\hline Technique & $\begin{array}{l}\text { Transmission } \\
\text { Mode }\end{array}$ & $\begin{array}{l}\text { Access } \\
\text { Mode }\end{array}$ & Complexity & Efficiency & $\begin{array}{c}\text { Types of } \\
\text { Interferenc } \\
\text { e }\end{array}$ \\
\hline Power Control & Downlink & Closed & Medium & High & Cross-tier \\
\hline Spectrum splitting & Downlink & Closed & Medium & High & $\begin{array}{c}\text { Cross-tierCo- } \\
\text { tier }\end{array}$ \\
\hline Fractional Frequency Reuse & Downlink & $\begin{array}{l}\text { Open and } \\
\text { Close }\end{array}$ & Medium & High & Cross-tier \\
\hline Cognitive Radio & Downlink & $\begin{array}{l}\text { Open and } \\
\text { Closed }\end{array}$ & Medium & Medium & Cross-tier \\
\hline Parallel Interference Cancellation & $\begin{array}{l}\text { Uplink/ } \\
\text { Downlink }\end{array}$ & $\begin{array}{l}\text { Open and } \\
\text { Closed }\end{array}$ & Medium & High & Cross-tier \\
\hline Successive Interference Cancellation & $\begin{array}{l}\text { Uplink/ } \\
\text { Downlink }\end{array}$ & $\begin{array}{l}\text { Open and } \\
\text { Closed }\end{array}$ & More & Medium & Cross-tier \\
\hline $\begin{array}{l}\text { Multiuser Successive Interference } \\
\text { Cancellation }\end{array}$ & Uplink & $\begin{array}{l}\text { Open and } \\
\text { Closed }\end{array}$ & Medium & High & Cross-tier \\
\hline Multiuser Detection & Uplink & Closed & More & Low & Cross-tier \\
\hline Particle Swarm Optimization & Downlink & Closed & Medium & Medium & $\begin{array}{c}\text { Co-tier/Cross- } \\
\text { tier }\end{array}$ \\
\hline Cuckoo Search & Downlink & Closed & More & High & Cross-tier \\
\hline Bat Algorithm & $\begin{array}{l}\text { Uplink/ } \\
\text { Downlink }\end{array}$ & Closed & Medium & High & $\begin{array}{c}\text { Co-tier/Cross } \\
\text { tier }\end{array}$ \\
\hline
\end{tabular}

combinatorial and continuous optimization problems. Following is the classes of SIA: Particle Swarm Optimization, Cuckoo search, artificial bee colony, Firefly algorithm, Ant colony optimization, Bat Algorithm.

I. Particle Swarm Optimization(PSO) based on social behavior of birds and it's a stochastic optimization method. PSO based on population-based search approach and depending on information sharing through population members. The search process is done using the probabilistic rules and combination of deterministic. Velocity and positon are the two vectors of the particle of each round that are uses in PSO algorithm. These vectors are updated on the basis of memory gained by the particle. In this method, the results quality depends on the value of scaling parameter.PSO algorithm determine the best serving Base stations(BSs) for users and records their location. So these maximize the network throughput [13].

II. Chaotic Bat Swarm Optimization (CBSO) is used for solving the different global optimization problem. CBSO algorithm includes into Bat Swarm Optimization (BSO) to minimize the premature coverage problem with the help of ergodicity and non-repetitious nature of chaotic functions of bat minimize the premature convergence problem. It is applied to high dimension problem and decreasing loudness in the signal.
III. Cuckoo Search is based on social rivet out the attention of their belligerent reproduction technique. Cuckoo Search Algorithm (CSA) based on stochastic global search metaheuristic methods. CSA is used for interference mitigation for the full frequency reuse. Base Station(BS) utilizes valid bandwidth which means full frequency reuse to connect without any interference from neighboring BS. Finding and allocating the proper bandwidth for all users the CSA is an efficient algorithm. The algorithm is canceled if given threshold value is less than the calculated interference on each subcarrier.

IV. Ant Colony Optimization(ACO) is based on the behavior of ants. This Algorithm uses for the throughtput system optimization which shows maximum and minimum throughput of the femtocell. Using this ACO we can discover the total rate of maximization but from the MUE interference not be considered[26].

V. Bat Algorithm(BA) uses for the resource allocation scheme on closed access mode in femtocell network. This algorithm focuses on mitigating the co-tier and cross-tier interference in macrocell-femtocell network by selecting the best resource blocks for FUE[17]. There are two important components of bat that affect the search characteristic of the algorithm: First is the diversification also called as exploration and other characteristic is intensification also called as exploitation. Diversification is to find solutions by 
exploring various different unknown spaces. This increses the convergence speed of algorithm.

\section{Future Direction For Research}

The heterogeneous network has resource allocation techniques which can be divided into: Splitting Frequency and Sharing Frequency. Sharing Frequency has subcarriers which shared among different layers that can maximize the resources. Splitting frequency is the resource is split to each layer. This frequency is widly used in the macrocellfemtocell network.Bat algorithm has the echolocation characteristics. They automatically adjust the wavelength of their transmitted pulses and adjust the rate of pulse transmission depending on the proximity of the target. The BA obtained the better result from when they dealing with lower dimensional optimization problem. BA coverage covered very quickly at an early stage and then the rate of convergence will go-slows down. The BA also find the better solution when there is a large number of population within a limited range. On the other hand, Differential Evolution solves the optimization problem over the continuous domain. DE gives the effective solution to problems without any expert knowledge or complex design. DE always order to keep track of the progress during the optimization process. Giving the direction and distance information from the population member to generate a result in adaptive technique with the better convergence properties. The DE has advantages of speed, robustness, and simple structure. DE always starts with the best solution and the continuous convergence is achieved in the higher dimensional optimization problem. DE gives better result when there is less number of population with no coverage limitation.

To improve BA characteristics for the higher dimensional problem, the standard bat algorithm is hybridized with differential evolution algorithm, in [23 ]. Hybrid Bat Algorithm(HBA) slightly improve the performance of standard bat algorithm, which can be used for mitigate the interference in femtocell and improve the area spectral efficiency.

\section{ConcLusion}

This survey paper gives basic overview of femtocell starts from history and background. It gives a motivation for research areas of femtocell which can be explored. It states the requirement, need and research efforts of the researcher in this area till date. This survey provides different Nature inspired algorithm to manage the co-tier and cross-tier interference in femtocell networks. The future research direction of femtocells has theoretically explained for researcher to improving spectral efficiency. The area spectral efficiency can be increased that is advantageous to both operators and subscribers.

\section{REFERENCES}

[1] http://www.qualcomm.com/solutions/wirelessnetworks/technologies/f emtocells.
[2] 3GPP, "Requirement for Envolved UTRA(E-UTRA) and Evolved UTRAN (EUTRAN)," TR 25.913, 3rd Generation Partnership Project(3GPP), Mar 2006.

[3] L. T. W. Ho and H. Claussen, "Effects of user -deployed, co-channel femtocells on the cell drop probability in a residential scenario," IEEE 18th International Symposium on Personal, Indoor and Mobile Radio Communication, pp.1-5,3-7 Sept.2007.

[4] R. Baines, "The nedd for WiMAX picocells and femtocells," WiMAX London, pp. 1-36, 25-26 April 2007.

[5] H. Su, L. Kuang and J. Lu, "Interference avoidance in OFDMA based femtocell network," IEEE youth Conference on Information, Computing and telecommunication, pp. 126-129, 20-21 Sept. 2009.

[6] M. Yavuz, F. Meshkati, S. Nanda, A. Pokharuyal,N. Johnson, B. Roghothaman, and A. Richardson,"Interference Management and performance analysis of UMTS/HSPA femtocells," IEEE commun. Mag. , vol. 47, no.9, pp. 102-109, sept. 2009.

[7] K. Han, Y. Choi, D. Kim, M. Na, S. Choi, "Optimization of femtocell network configuration under interference constraints," 7 th International Symposium on Modeling and Optimization in Mobile, Ad Hoc, and Wireless Networks, pp. 1-7, 23-27 Jun.2009.

[8] A. K. Elhakeen, R. D. Girolamo, I.B. Bdira, and M. Talla, "Delay and throughput characteristics of cdma, tdma and hybrid networks for multipath faded data transmission channels," IEEE J. Se. Areas Commun., vol 12, no.4, pp.622-637, May 1994

[9] T. Kim and T. Lee, "Throughput enhancement if macro and femto networks by frequency reuse and pilot sensing," in performance, computing and communication Conference, IPCCC 2008. IEEE International, pp. 390-394, IEEE, 2008

[10] P. Frenger, P. Orten and T. Ottosson, "Code spread cdma with interference cancellation," IEEE J. Sel. Areas Commun., vol. 17, no. 12, pp. 2090-2095, 1999.

[11] J. G. Andrews, "Interference Cancellation for cellular system: A contemporary overview," IEEE Wireless Commun., vol 2, no. 3, pp. 19-29, April, 2005.

[12] M. Honig, U. Madhow, and S. Verdu, "Blind adaptive multiuser detection," IEEE Trans. Inf. Theory, vol. 41, no. 4, pp. 944-960, July 1995.

[13] R. Estrada, H. Otrol, and Z. Dziong, Resource allocation model based on particle swarm optimization for OFDMA macro-femtocell networks, IEEE ANTS,2013.

[14] A. Rezaee Jordehi, Chaotic bat swarm optimization, Elsever, Applied Soft Computing, 2014.

[15] Motea Al-omari, Abd Rahman Ramli, A. Sali and Raja Syamsul Azmir, A Femtocell cross-tier interference mitigation technique in OFDMA LTE system: A cuckoo search based approach, Indian Journal of Science and Technology, Vol9(2), DOI: 10.17485/ijst/2016/v9i2/80490, January 2016.

[16] J. Zhou, X. She, L. Chen, and H. Otsuka, Qos guaranteed radio resource allocation scheme using genetic algorithm for OFDMA. In Communications and Networking in china (CHINACOM), 2011 6th International ICST Conference on, pages 594-599. IEEE, 2011.

[17] Selim Yilmaza, Ecir U. Kucuksille, A new modification approach on bat algorithm for solving optimization problems, Applied Soft Computing 28 (2015) 259-275, Elsevier, 2014.

[18] Ahmad Rezaee Jordehi, Chaotic bat swarm optimization, Articke in apllied soft computing. October 2014

[19] Xin She Yang, A new metaheuristic bat inspired algorithm, April 2010.

[20] Rebeca Estrada, Hali Otrok et el. Resource allocation model based on particle swarm optimization for OFDMA macro femtocell networks, IEEE ANTS,2013.

[21] Hanaa Marshod, Hadi Otrok, et el., Resource allocation in macrocell femtocell network using genetice algorithm, IEEE 8th International conference on wireless and mobile computing, networking and communication (WiMob), 2008.

[22] Zhuo Li, Song Guo, et. el., A particle swarm optimization algorithm for resource allocation in femtocell networks, IEEE wireless communication and networking conference: MAC \& Cross-layer design, 2012.

[23] Iztok Fister, Xin She Yang, et. el., A hybrid bat algorithm, 5 june 2013.

[24] Vu Truong Vu, A coparison of particle swarm optimization and differential evolution, International Journal on soft computing vol 3 , August 2012.

[25] Xin She Yang, Bat algorithm for multiobjective optimization, 29 march 2012 .

[26] D. Liu, H. Zheng, and X. Wen, The sub-channel allocation algorithm in femtocell networks based on ant colony optimization, IEEE, 2013. 